\title{
De la plante aux effets biologiques de l'extrait : quand la démarche scientifique éclaire les usages
}

\section{From the Plant to the Biological Effects of the Extract: When the Scientific Approach Sheds Light on Uses}

\author{
I. Guinobert · V. Bardot · M. Dubourdeaux
}

(C) Lavoisier SAS 2019

Résumé L'intérêt porté aux plantes médicinales a considérablement été renouvelé ces dernières années. Cependant, les données fiables sur les extraits de plantes commercialisés sont souvent inexistantes. Une partie du savoir-faire industriel du groupe PiLeJe est axé sur la production d'extraits de plantes dont le profil phytochimique est proche de celui du totum. Le laboratoire s'efforce d'apporter des preuves scientifiques sur la qualité, l'usage et l'intérêt des extraits de plantes produits. Cela nécessite de recourir à des procédés industriels adaptés, permettant d'extraire et de restituer intacte la majorité des composants de la plante et d'améliorer de façon continue la filière et la production des extraits. De nombreux efforts sont également fournis pour standardiser et caractériser chaque extrait de plante produit et associer au profil phytochimique établi des propriétés et des effets biologiques.

Mots clés Extrait de plante · Extraction · Lixiviation · Standardisation $\cdot$ Caractérisation $\cdot$ Totum

\begin{abstract}
Interest in medicinal plants has been considerably renewed in recent years. However, reliable data on marketed plant extracts are often lacking. Part of the PiLeJe Group's industrial expertise is focused on the production of plant extracts with a phytochemical profile similar to that of plant totum. The laboratory strives to provide scientific evidence on the quality, use and interest of the plant extracts produced. This requires the use of appropriate industrial processes to extract and restore most of the plant components intact, and to continuously improve procedures and the production of extracts. Many efforts are also being made to standardize and characterize each plant extract produced and to associate
\end{abstract}

I. Guinobert $(\bowtie) \cdot$ V. Bardot $\cdot$ M. Dubourdeaux

Groupe PiLeJe, 37, quai de Grenelle,

F-75015 Paris cedex 15, France

e-mail : i.guinobert@pileje.com

Naturopôle, Les Tiolans,

F-03800 Saint-Bonnet-de-Rochefort, France biological properties and effects with the established phytochemical profile.

Keywords Plant extract · Extraction - Lixiviation Standardization $\cdot$ Characterization $\cdot$ Totum

\section{Introduction}

Les extraits de plantes sont utilisés en médecine depuis des siècles. Aujourd'hui, alors que certains extraits de plantes bénéficient à la fois de données précliniques et cliniques probantes, bien des professionnels de santé et patients restent critiques quant à l'efficacité de la phytothérapie. La raison est la présence sur le marché d'extraits de plantes de qualité très variable, non standardisés et mal caractérisés, et le manque de données fiables sur leurs bienfaits et leur tolérance, éléments clés dans une approche de médecine fondée sur les preuves. Néanmoins, l'intérêt porté aux plantes médicinales a considérablement été renouvelé ces dernières années en raison notamment d'une méfiance grandissante à l'égard des médicaments et de l'engouement pour des solutions de santé naturelles. Pour répondre aux interrogations et aux attentes des professionnels de santé et des patients, il est devenu incontournable d'améliorer les connaissances scientifiques sur les extraits de plantes, c'est-à-dire de les caractériser d'un point de vue phytochimique pour montrer leurs spécificités moléculaires et d'apporter des preuves de leurs bienfaits et de leur innocuité.

L'un des concepts majeurs en phytothérapie est celui de synergie selon lequel l'activité d'un extrait d'une plante est plus que la somme des effets de ses constituants $[1,2]$. La pluralité des actifs permet d'agir sur des cibles multiples et d'inhiber, par exemple, différentes étapes des mécanismes infectieux, d'interagir avec les micro-organismes, de courtcircuiter les mécanismes de résistance ou encore d'empêcher des effets secondaires [1]. Les synergies sont non seulement liées à la diversité des actifs présents mais également à 
l'action adjuvante de certaines molécules auxquelles l'action médicinale n'est pourtant pas attribuée. Ces molécules utiles peuvent accroître la solubilité des actifs et donc leur biodisponibilité [1]. Elles peuvent aussi favoriser le transport des actifs, les protéger contre l'action métabolique des enzymes tissulaires et donc leur permettre d'atteindre intègres leurs sites d'action [3]. L'ensemble des molécules actives et utiles de la plante constituent le totum. L'association complexe de toutes ses molécules confère à la plante des propriétés médicinales parfois différentes de celles obtenues par seulement certains de ses composants issus d'une extraction sélective, ce qui renforce la perception que le totum a quelque chose de particulier à offrir. En axant une partie de sa recherche et de son savoir-faire industriel sur la production d'extraits de plantes dont le profil phytochimique est proche de celui du totum, le groupe PiLeJe s'inscrit parfaitement dans ce cadre conceptuel. La production de ce type d'extraits nécessite de recourir à des procédés industriels adaptés, permettant d'extraire et de restituer intacte la majorité des composants de la plante. En outre, le profil phytochimique et donc les effets d'un extrait de plante sont dépendants de la qualité de la matière première utilisée, la plante, de sa provenance (Fig. 1), de la saison à laquelle elle a été récoltée mais aussi de son état (fraîche ou sèche). En effet, la proximité du profil phytochimique d'un extrait par rapport à celui de la plante, ou l'équivalence entre des extraits de plantes provenant de différentes régions du globe, issues de récoltes ou de procédés distincts n'est garanti ni par la quantité de plantes mise en œuvre lors de la fabrication ni par la quantité de matière sèche dans le produit fini. De nombreuses études publiées dans la littérature ont rapporté des données très variables sur la caractérisation, les propriétés et les effets biologiques de différents extraits d'une même plante (Jean et al., Azmir et al., Andrade et al., Dimpfel et al. et Zengin et al. [4-8]).

Dans la perspective d'apporter des données et des preuves scientifiques sur la qualité, l'usage et l'intérêt des extraits de plantes produits, de nombreux efforts sont fournis par le groupe PiLeJe pour :

- améliorer de façon continue la filière et la production des extraits ;

- standardiser et caractériser chaque extrait de plante ;

- associer au profil phytochimique établi des propriétés et des effets biologiques (Fig. 2).

\section{Amélioration continue de la filière et de la production des extraits de plantes}

La composition des extraits de plantes dépend en premier lieu de la qualité des plantes d'origine. L'identification exacte des espèces est une étape primordiale permettant de répondre aux exigences réglementaires et d'assurer la sécu-

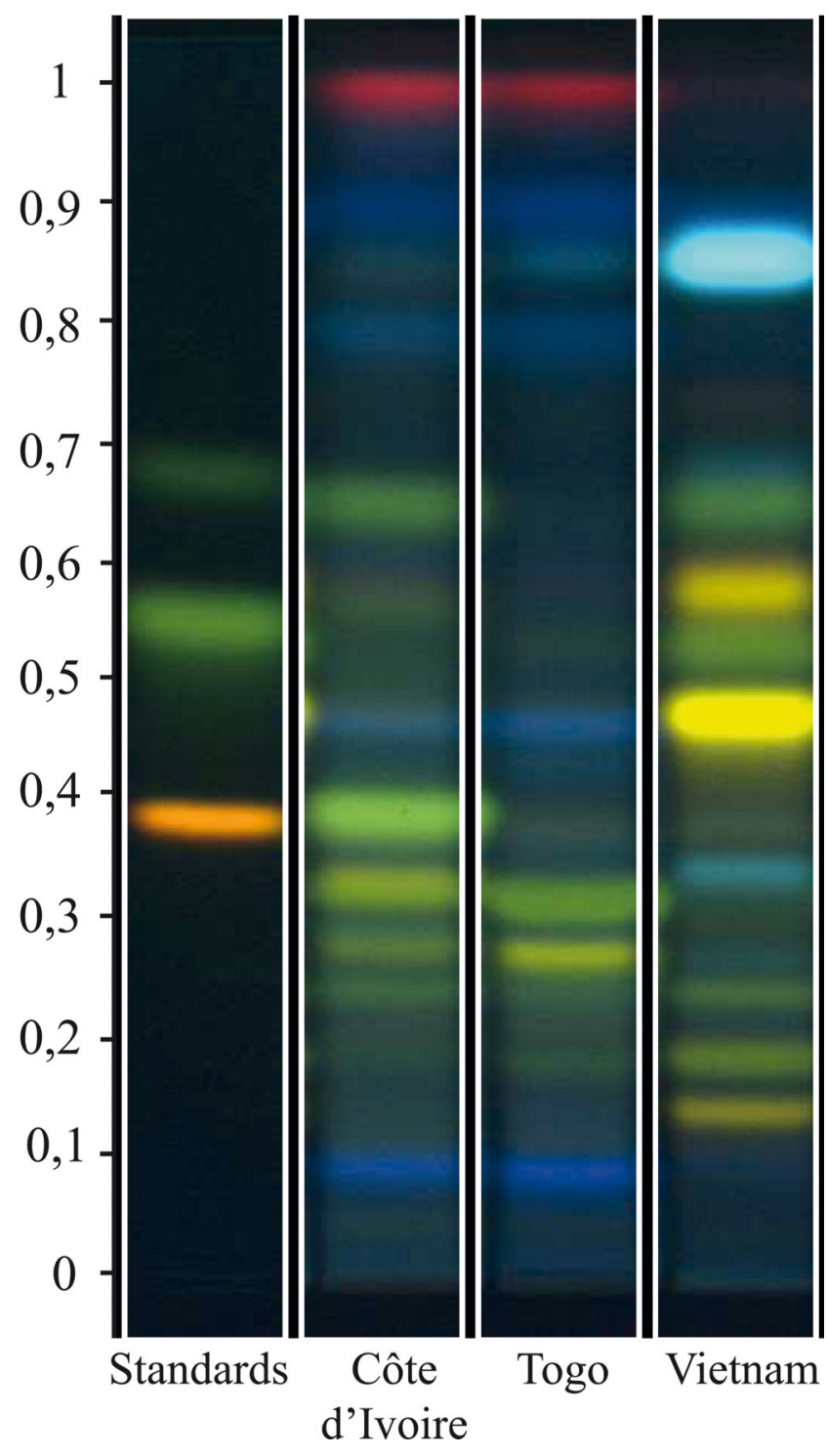

Fig. 1 Variabilité du profil phytochimique du Desmodium adscendens (Sw.) Dc. selon son origine géographique

rité et la qualité des extraits. L'étude des caractères phénotypiques, la conformité aux monographies des Pharmacopées lorsque celles-ci existent et la réalisation de planches d'herbier sont indispensables pour répertorier les espèces d'intérêt, répondre aux exigences pharmaceutiques et éviter les risques de confusion. La maîtrise de la sélection des plantes avec une haute teneur en actifs, une matière végétale de qualité biologique à la traçabilité rigoureuse et répondant à un cahier des charges strict que les producteurs s'engagent à respecter est exigée, et l'utilisation de plantes fraîches permettant de préserver un maximum d'actifs est indispensable. En l'occurrence, le moment de récolte, le niveau de maturité, la hauteur de coupe, les précautions pour ne pas abîmer le reste de la partie aérienne de la plante lors de la coupe et le délai entre la coupe et la congélation sont strictement 


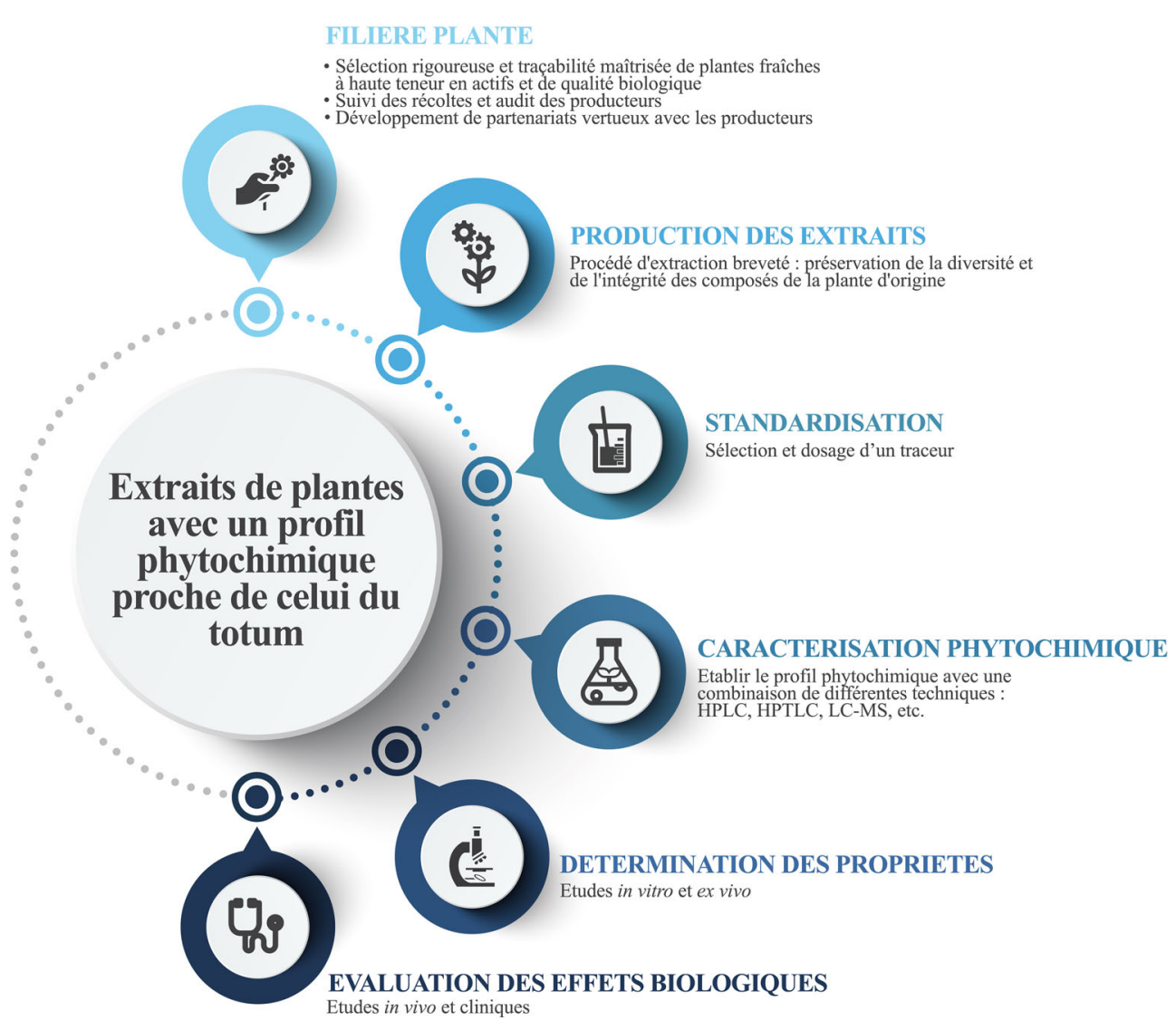

Fig. 2 Expertise végétale : de la plante aux effets biologiques de l'extrait

spécifiés - des plantes fragiles comme l'orthosiphon, sensible à l'oxydation, doivent être congelées le jour même de leur récolte. Des audits et des échantillonnages périodiques sont effectués pour s'assurer du respect des bonnes pratiques de culture et de récolte, de la teneur des plantes en actifs et de l'absence de contaminants. De nombreux contrôles supplémentaires sont aussi réalisés avant la libération des lots pour permettre leur utilisation pour la fabrication des extraits. Par ailleurs, conformément à ses valeurs d'écoresponsabilité, le groupe PiLeJe a aussi développé des partenariats vertueux avec ses producteurs dans une démarche de développement durable qui assure la pérennité des ressources naturelles (plan de gestion des cultures, certification ECOCERT, accroissement de la matière organique du sol, agroforesterie, etc.) et qui favorise le développement économique des populations locales avec respect des savoir-faire et des traditions lorsque les plantes proviennent de régions lointaines.

En plus de la qualité des plantes d'origine, la composition des extraits dépend étroitement du procédé de production, des équipements, du(des) solvant(s) et des conditions de réalisation.

Une innovation majeure dans ce domaine est celle du procédé Phytostandard $^{\circledR}$ [9] qui préserve de manière optimale la diversité et l'intégrité des composés de la matière végétale brute, garantissant ainsi un profil phytochimique des extraits au plus proche de celui de la plante fraîche. En l'occurrence, le profil d'un extrait obtenu par ce procédé est bien plus large que celui des extraits obtenus par extraction hydroalcoolique unique [10]. Une étude comparative a montré que ces extraits ont systématiquement une plus grande diversité moléculaire que les teintures mères correspondantes, et qu'ils sont aussi plus concentrés en actifs d'un facteur dépassant 3 pour certaines plantes telles que la mélisse et l'artichaut [11]. Le procédé

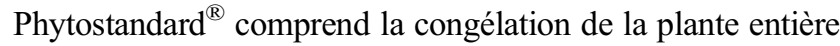
ou de ses parties actives, le broyage, la lixiviation de la plante par un solvant organique miscible à l'eau, la réunion des solutions extractives de lixiviation après leur concentration, ainsi que des limites de température pour réaliser ces opérations. Selon les plantes et leurs parties d'intérêt, l'expertise industrielle du groupe PiLeJe a permis de spécifier des conditions adaptées de réalisation de ces opérations et d'ajouter des opérations quand c'est nécessaire.

\section{Standardisation et caractérisation analytique des extraits de plantes}

La sélection et l'utilisation d'un traceur permettent de standardiser les extraits fluides, commercialisés sous la dénomination EPS pour extraits fluides de plantes fraîches 
standardisés. Un traceur est une molécule ou une famille de molécules dont la présence et le dosage sont caractéristiques de l'extrait et de sa qualité. Il peut s'agir d'un actif ou d'une molécule représentative du profil phytochimique du totum présentant un intérêt pour le contrôle, pour sélectionner une espèce, une origine géographique ou la partie de plante qui présente le plus d'intérêt. Enfin, un traceur permet d'étudier la stabilité des extraits après leur fabrication, et donc d'en déterminer la date de péremption, et de valider la bonne conservation d'un lot de production. Les extraits fluides produits par le groupe PiLeJe sont standardisés en concentration de traceur sur la base des données bibliographiques, de la teneur de la plante fraîche ou du rendement d'extraction, et de manière à être conforme à la monographie des extraits fluides de la Pharmacopée européenne (statut matières premières à usage pharmaceutique [MPUP]).

Les extraits de plantes sont caractérisés en utilisant et combinant différentes méthodes analytiques fondées sur des techniques modernes, comme la chromatographie sur couche mince haute performance (HPTLC ; [12]), la chromatographie en phase liquide à haute performance (HPLC) et l'ultra-(U)HPLC, et la chromatographie en phase liquide couplée à la spectrométrie de masse (LC-MS et LC-MS ${ }^{2}$ ).

La combinaison de ces différentes techniques constitue une approche globale essentielle pour déterminer la compo- sition des extraits de plantes, sélectionner une origine locale ou une variété particulière, adapter les conditions d'extraction pour optimiser la composition des extraits, comparer les profils phytochimiques d'un extrait et de la matière brute végétale, de la matière fraîche et de la matière sèche, et comparer différents lots pour évaluer la reproductibilité du procédé industriel. La connaissance plus approfondie des profils phytochimiques qui en découlent permet de renforcer les critères de qualité à de nombreux niveaux (sélection des variétés végétales et de leur origine, vérification de la stabilité des produits, etc.), de mieux comprendre et de documenter les propriétés et les effets biologiques mais également de garantir leur reproductibilité. Plusieurs extraits de plantes commercialisés ont déjà été caractérisés, avec la détection de différentes molécules ou familles de molécules d'intérêt (Tableau 1).

\section{Association du profil phytochimique à des propriétés et/ou des effets biologiques}

Une fois les extraits de plantes caractérisés, le groupe PiLeJe réalise les études nécessaires afin de mettre en évidence les propriétés in vitro ou ex vivo de ces mêmes extraits et leurs effets biologiques.

Tableau 1 Molécules ou familles de molécules identifiées par HPTLC et LC/MS, LC/MS ${ }^{2}$ dans différents extraits de plantes fraîches standardisés

\begin{tabular}{|c|c|c|}
\hline Extrait hydroéthanolique non glycériné & Molécules ou familles de molécules détectées & Références \\
\hline $\begin{array}{l}\text { Astragalus mongholicus — Extrait de racines } \\
\text { fraîches d'astragale comparé à la matière brute }\end{array}$ & $\begin{array}{l}\text { Profils similaires avec de plus fortes concentrations } \\
\text { en astragalosides IV (glycoside triterpène) } \\
\text { et formononétine (flavonoïde) dans l'EPS } \\
\text { (HPTLC et LC/MS) }\end{array}$ & {$[13,14]$} \\
\hline $\begin{array}{l}\text { Cupressus sempervirens L. - Extrait de cônes frais } \\
\text { de cyprès }\end{array}$ & $\begin{array}{l}\text { Catéchine, épicatéchine, tanins de type } \\
\text { proanthocyanidines, acides aminés, glucides } \\
\text { et flavonoïdes (HPTLC et LC/MS, LC/MS }{ }^{2} \text { ) }\end{array}$ & {$[15]$} \\
\hline $\begin{array}{l}\text { Juglans regia L. - Extrait de feuilles fraîches } \\
\text { de noyer }\end{array}$ & $\begin{array}{l}\text { Flavonoïdes (dérivés de quercétine, myricétine, kaempférol } \\
\text { et taxifoline) et acides hydroxycinnamiques (acide } \\
\text { chlorogénique, acide néochlorogénique, } \\
\text { et acides 3- et 4-p-coumaroylquinique) }\end{array}$ & {$[16]$} \\
\hline $\begin{array}{l}\text { Melissa officinalis L. - Extrait de feuilles fraîches } \\
\text { de mélisse }\end{array}$ & $\begin{array}{l}\text { Acide rosmarinique, dérivés d'acide rosmarinique } \\
\text { et caféique (Danshensu, acide rosmarinique } \\
\text { 3'-O-[8"-Z-Caffeoyl], caféate d'éthyle), } \\
\text { flavonoïdes (lutéoline 3'-O- } \beta \text {-D-glucuronide) }\end{array}$ & Données internes \\
\hline $\begin{array}{l}\text { Rhodiola rosea } \mathrm{L} \text {. - Extrait fluide glycériné } \\
\text { et standardisé de racines fraîches de rhodiole }\end{array}$ & $\begin{array}{l}\text { Salidroside et rosavine, glycosides monoterpéniques } \\
\text { (rhodiolosides et rosidrine), dérivés de phénylpropane } \\
\text { (rosarine et rosine) et flavonoïdes (kaempférol } \\
\text { et rhodaline) }\end{array}$ & [17] \\
\hline $\begin{array}{l}\text { Valeriana officinalis L. - Extrait de racines } \\
\text { fraîches de valériane }\end{array}$ & $\begin{array}{l}\text { Acide hydroxyvalérénique, acide acétoxyvalérénique, } \\
\text { acide valérénique }\end{array}$ & {$[18]$} \\
\hline
\end{tabular}




\section{Astragalus mongholicus Bunge}

Un extrait de racines fraîches d'astragale (Astragalus mongholicus Bunge ; extrait hydroéthanolique non glycériné) a induit in vitro un accroissement de la longueur des télomères via une augmentation de l'activité de la télomérase [13]. Après 72 heures d'exposition à l'extrait $(1 \mu \mathrm{g} / \mathrm{ml})$, la proportion de lymphocytes à télomères courts était moins importante pour certains donneurs. Ce résultat est susceptible d'être lié à la forte teneur en flavonoïdes et en astragalosides de l'extrait mise en évidence par l'analyse phytochimique. Ces résultats, compatibles avec le fait que l'astragale ou certains de ces actifs isolés soient connus pour stimuler l'activité de la télomérase avec un impact bénéfique sur la longueur des télomères $[19,20]$, renforcent l'intérêt d'évaluer l'effet in vivo d'une supplémentation avec cet extrait pour la prise en charge de maladies liées à l'âge.

\section{Cupressus sempervirens $\mathrm{L}$.}

Un extrait de cônes frais de cyprès (Cupressus sempervirens L. ; extrait hydroéthanolique non glycériné), précédemment caractérisé d'un point de vue phytochimique, a eu une activité virucide sur des virus humains et des virus bovins [15]. À $40 \%(\mathrm{v} / \mathrm{v})$, au contact des virus pendant 60 minutes à $37^{\circ}$ $\mathrm{C}$, l'extrait a eu une activité virucide sur le coronavirus, le virus para-influenza de type 3 , le virus respiratoire syncytial bovin et le rotavirus bovin. À $80 \%$ (v/v), l'extrait a eu une activité virucide sur le virus influenza A-H1N1, le rhinovirus et l'herpès virus bovin de type 1 . La caractérisation de cet extrait, en particulier la présence de tanins, rapprochée aux connaissances disponibles dans la littérature a permis de formuler des hypothèses sur les mécanismes conduisant à la perte du pouvoir infectieux des virus. Ces résultats encouragent l'évaluation des effets de l'extrait de cyprès en utilisation prophylactique et thérapeutique complémentaire en médecine humaine et vétérinaire.

\section{Juglans regia $\mathbf{L}$.}

L'extrait de feuilles fraîches de noyer (Juglans regia L. ; EPS Noyer) a eu une activité antidiarrhéique et a diminué la douleur dans un modèle de diarrhée induite chez le rat par de l'huile de ricin [16]. Le retard dose-dépendant de l'apparition de la diarrhée induite, la diminution du nombre et du poids des fèces diarrhéiques, de leur teneur en eau, la moindre perte de masse corporelle et la diminution de la douleur suggèrent que cet extrait peut constituer une alternative intéressante au lopéramide - traitement antimotilité standard qui n'a pas diminué la douleur dans le modèle utilisé. La connaissance du profil moléculaire associée aux connaissances disponibles dans la littérature a permis d'émettre des hypothèses sur les mécanismes impliqués et les synergies potentielles entre molécules, notamment sur l'inflammation mucosale, la perte hydroélectrolytique, l'activité spasmodique et la douleur. Par exemple, les flavonoïdes et les acides hydroxycinnamiques détectés dans l'extrait pourraient expliquer les effets observés.

\section{Melissa officinalis L.}

L'extrait de feuilles fraîches de mélisse (Melissa officinalis L. ; extrait hydroéthanolique non glycériné) a eu une activité antispasmodique ex vivo sur le segment jéjunal de l'appareil gastro-intestinal de la souris, aussi bien en termes de fréquence des contractions phasiques en conditions basales qu'en termes de niveau de contraction isométrique en conditions de précontraction tissulaire muscarinergique [21]. Au vu de ces résultats, il serait intéressant d'évaluer l'effet d'une supplémentation avec cet extrait sur les troubles de la motricité digestive et les symptômes associés.

\section{Rhodiola rosea $\mathrm{L}$.}

La complémentation quotidienne avec un extrait de racines fraîches de rhodiole (Rhodiola rosea L. ; EPS Rhodiole) a diminué le taux sanguin de corticostérone chez des souris soumises à un stress aigu modéré (dix minutes en champ ouvert puis cinq minutes en labyrinthe en croix surélevé : [17]). Cette diminution était associée à un effet modulateur de l'expression d'un ensemble de gènes impliqués dans l'activation du système endocrinien qui contrôle notamment les réponses au stress. L'effet modulateur dépendait des structures cérébrales, hippocampe, cortex préfrontal et amygdale. Ces résultats sont compatibles avec un effet potentiel sur la résistance au stress pour lequel l'usage de la rhodiole est recommandé [22-24] et suggèrent que cet extrait pourrait constituer une option intéressante à explorer pour diminuer les effets néfastes d'une réponse au cortisol excessive due au stress chez l'homme.

\section{Valeriana officinalis $\mathbf{L}$.}

L'extrait de racines fraîches de valériane (Valeriana officinalis L. ; EPS Valériane) a eu une activité myorelaxante sur le muscle squelettique de la souris [18]. En effet, la supplémentation par voie orale avec une seule dose de l'extrait de valériane a diminué la force musculaire des souris $(18 \%$ de force en moins au test d'agrippement 30 minutes après l'administration d'une dose de $2 \mathrm{~g} / \mathrm{kg}$ ). Contrairement à l'administration de tétrazépam (molécule de référence ; $10 \mathrm{mg} / \mathrm{kg}$ ), l'extrait de valériane n'a pas eu d'impact sur l'endurance ni sur le tonus neuromusculaire (test de suspension à un fil). Les résultats obtenus avec cet extrait montrent un avantage sur le maintien du tonus neuromusculaire et de l'endurance. L'effet myorelaxant de cet extrait dans le traitement des 
contractures musculaires ainsi que l'intérêt de son usage pour éviter ou limiter les effets indésirables des myorelaxants standard tels que la perte de vigilance et l'augmentation du risque de chute devront être confirmés.

En plus des études réalisées sur différents modèles animaux, deux études cliniques ayant montré des effets biologiques d'extraits de plantes sous forme de comprimés ont été conduites. La complémentation quotidienne pendant un mois avec une combinaison d'extraits secs obtenus selon le procédé Phytostandard ${ }^{\circledR}$ de parties aériennes fleuries frâ̂ches d'eschscholtzia (Eschscholtzia californica Cham.) et de racines fraîches de valériane (Valeriana officinalis L.) a eu un effet bénéfique sur les caractéristiques de l'insomnie et également sur l'anxiété chez les patients souffrant d'insomnie liée à l'anxiété [25]. Les résultats de cette étude conduite chez 36 patients sont compatibles avec les bienfaits hypnotiques, anxiolytiques et sédatifs et les préconisations d'usage pour traiter les troubles du sommeil et les symptômes légers de stress de l'eschscholtzia et de la valériane [26,27], et suggèrent qu'une telle combinaison pourrait être intéressante pour la prise en charge de l'insomnie liée à l'anxiété.

Dans une autre étude, la complémentation quotidienne pendant six semaines avec une combinaison d'un extrait sec obtenu selon le procédé Phytostandard ${ }^{\circledR}$ de racines de rhodiole (Rhodiola rosea L.) et d'un extrait sec de stigmates de safran (Crocus sativus L.) a eu un effet antidépresseur et antianxiété chez les patients souffrant de dépression légère à modérée [28]. Les résultats de cette étude conduite par des généralistes chez 45 patients sont compatibles avec les usages traditionnels de la rhodiole, pour ses bienfaits sur l'équilibre nerveux et la gestion du stress [29,30], et du safran, pour ses vertus concernant l'amélioration des symptômes liés à la dépression [31-33]. Ils suggèrent qu'une telle combinaison constitue une alternative intéressante aux traitements antidépresseurs conventionnels dont l'efficacité chez des patients souffrant de dépression légère à modérée est controversée, et l'observance est impactée par des effets indésirables substantiels [34].

\section{Conclusion}

Le groupe PiLeJe a mis en place de nombreuses actions pour maitriser la filière et la production de ses extraits, pour standardiser et caractériser les extraits de plantes obtenus et associer à leur profil phytochimique des propriétés et des effets biologiques. L'identification de la plante, la standardisation et la détermination du profil phytochimique sont trois éléments clés indissociables pour pouvoir lier un extrait de plante donné à des effets biologiques spécifiques et si l'on veut être reproductible. Les études in vitro et ex vivo aident à mettre en évidence des propriétés des extraits de plantes, et les études in vivo permettent d'en démontrer les bénéfices pour le traitement et/ou la prévention de diverses pathologies et troubles associés. Cette stratégie contribue à démontrer la place de choix que peut avoir ce type d'extraits dans l'arsenal thérapeutique et naturel que propose la phytothérapie.

Remerciements Nous remercions Jean-Philippe Petit (rédacteur indépendant), Claude Blondeau et Aurélie Berlin (PiLeJe Laboratoire) pour leur assistance rédactionnelle et éditoriale

Liens d'intérêts : les auteurs déclarent être salariés du Groupe PiLèJe.

\section{Références}

1. Wagner H, Ulrich-Merzenich G (2009) Synergy research: approaching a new generation of phytopharmaceuticals. Phytomedicine 16:97-110

2. Williamson EM (2001) Synergy and other interactions in phytomedicines. Phytomedicine 8:401-9

3. Gilbert B, Ferreira Alves L (2003) Synergy in plant medicines. Curr Med Chem 10:13-20

4. Jean D, Pouligon M, Henriot AC (2006) Pharmacological activity of three commercial Hypericum perforatum preparations in mice. Phytother Res 20:653-4

5. Azmir J, Zaidul ISM, Rahman MM, et al (2013) Techniques for extraction of bioactive compounds from plant materials: a review. J Food Eng 117:426-36

6. Andrade JM, Faustino C, Garcia C, et al (2018) Rosmarinus officinalis L.: an update review of its phytochemistry and biological activity. Future Sci OA 4:FSO283

7. Dimpfel W, Schombert L, Panossian AG (2018) Assessing the quality and potential efficacy of commercial extracts of Rhodiola rosea $\mathrm{L}$. by analyzing the salidroside and rosavin content and the electrophysiological activity in hippocampal long-term potentiation, a synaptic model of memory. Front Pharmacol 9:425

8. Zengin G, Senkardes I, Mollica A, et al (2018) New insights into the in vitro biological effects, in silico docking and chemical profile of clary sage - Salvia sclarea L. Comput Biol Chem 75:111-9

9. Institut des substances végétales (2002) Procédé de préparation d'extraits végétaux, extraits ainsi obtenus et leur utilisation. Jean Daniel. Brevet WO 01/56584A1

10. Dessouroux A, Seyrig C, Leclerc C (2011) Point sur la qualité des extraits fluides glycérinés de plantes fraîches standardisés (EPS) et leur intérêt pharmacologique. Phytothérapie 9:249-54

11. Guilbot A, Dessouroux A, Jean D, et al (2013) Comparaison des profils chromatographiques d'extraits fluides de plantes fraîches standardisés et glycérinés (EPS) versus teintures mères : différences d'intensité et de diversité. Phytothérapie 11:301-5

12. Reich E, Schibli A (2007) High-performance thin-layer chromatography for the analysis of medicinal plants. Thieme Medical, New York, NY

13. Guinobert I, Bardot V, Cotte C, et al (2017) Astragalus mongholicus hydroethanolic root extract: HPTLC characterization and effects on telomere elongation. Planta Med International Open 4:S1-S202, Basel, Switzerland

14. Berthomier L, Bardot V, Dubourdeaux M, et al (2017) Fingerprint of an Astragalus mongholicus extract by HTPLC and LCMS and quantification of formononetin with densitometric HPTLC. International Symposium on High Performance ThinLayer Chromatography (4-8 July), Berlin, Germany 
15. Guinobert I, Bardot V, Berthomier L, et al (2018) Activité virucide in vitro d'un extrait de cyprès sur des virus humains et bovins. Phytothérapie 16:281-9

16. Holowacz S, Blondeau C, Guinobert I, et al (2016) Anti-diarrheal and anti-nociceptive effects of a hydroethanolic leaf extract of walnut in rats. Med Aromat Plants (Los Angel) 5:268

17. Guinobert I, Dinel AL, Joffre C, et al (2018) Administration of a standardized extract of Rhodiola rosea in mice reduced acute mild stress-induced corticosterone release and altered stressresponsive gene expression. Poster présenté au 22nd International Phytopharm Congress (25-27 June), Horgen and ZHAW Wädenswil, Switzerland

18. Caudal D, Guinobert I, Lafoux A, et al (2017) Skeletal muscle relaxant effect of a standardized extract of Valeriana officinalis L. after acute administration in mice. J Tradit Complement Med $8: 335-40$

19. Molgora B, Bateman R, Sweeney G, et al (2013) Functional assessment of pharmacological telomerase activators in human T cells. Cells 2:57-66

20. Jäger K, Walter M (2016) Therapeutic targeting of telomerase. Genes (Basel) 7:39

21. Aubert P, Guinobert I, Blondeau C, et al (2019) Basal and Spasmolytic Effects of a Hydroethanolic Leaf Extract of Melissa officinalis L. on Intestinal Motility: An Ex Vivo Study. J Med Food. doi: 10.1089/jmf.2018.0154 - [In press]

22. Anghelescu IG, Edwards D, Seifritz E, et al (2018) Stress management and the role of Rhodiola rosea: a review. Int J Psych Clin Pract 11:1-11 doi:10.1080/13651501.2017.1417442

23. Palmeri A, Mammana L, Tropea MR, et al (2016) Salidroside, a bioactive compound of Rhodiola rosea, ameliorates memory and emotional behavior in adult mice. J Alzheimer's Dis 52:65-75

24. Mattioli, L, Funari C, Perfumi M (2009) Effects of Rhodiola rosea $\mathrm{L}$. extract on behavioural and physiological alterations induced by chronic mild stress in female rats. J Psychopharmacol 23:130-42
25. Ait Abdellah S, Berlin A, Blondeau C, et al (2018) A combination of Eschscholtzia californica Cham. and Valeriana Officinalis L. extracts for adjustment insomnia: a prospective observational study. J Tradit Complement Med. [In press] https://www.sciencedirect.com/science/article/pii/S2225411019302391

26. Leach MJ, Page AT (2015) Herbal medicine for insomnia: a systematic review and meta-analysis. Sleep Med Rev 24:1-12

27. European Medicines Agency (2015) European Union herbal monograph on Eschscholzia californica Cham., herba. http:// www.ema.europa.eu/docs/en_GB/document_library/Herbal_-_ HMPC_assessment_report/2015/05/WC500186550.pdf. Accès 25 janvier 2019

28. Bangratz M, Ait Abdellah S, Berlin A, et al (2018) A preliminary assessment of a combination of rhodiola and saffron in the management of mild-moderate depression. Neuropsychiatr Dis Treat 14:1821-9

29. Amsterdam JD, Panossian AG (2016) Rhodiola rosea L. as a putative botanical antidepressant. Phytomedicine 23:770-83

30. Darbinyan V, Aslanyan G, Amroyan E, et al (2007) Clinical trial of Rhodiola rosea L. extract SHR-5 in the treatment of mild to moderate depression. Nord J Psychiatry 61:343-8

31. Hausenblas HA, Saha D, Dubyak PJ, et al (2013) Saffron (Crocus sativus L.) and major depressive disorder: a meta-analysis of randomised clinical trials. J Integr Med 11:377-83

32. Mazidi M, Shemshian M, Mousavi SH, et al (2016) A doubleblind, randomized and placebo-controlled trial of saffron (Crocus sativus L.) in the treatment of anxiety and depression. J Compl Integr Med 13:195-9

33. Shafiee M, Arekhi S, Omranzadeh A, et al (2018) Saffron in the treatment of depression, anxiety and other mental disorders: current evidence and potential mechanisms of action. J Affect Disord 227:330-7

34. Zimmermann TM, Clouth J, Elosge M, et al (2013) Patient preferences for outcomes of depression treatment in Germany: a choicebased conjoint analysis study. J Affect Disord 148:210-9 